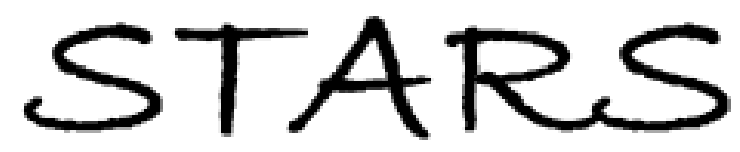

University of Central Florida

STARS

Faculty Bibliography 2000s

Faculty Bibliography

$1-1-2009$

\title{
Extremely low scattering cross section of a perforated silver film
}

Haining Wang

University of Central Florida

Shengli Zou

University of Central Florida

Find similar works at: https://stars.library.ucf.edu/facultybib2000

University of Central Florida Libraries http://library.ucf.edu

This Article is brought to you for free and open access by the Faculty Bibliography at STARS. It has been accepted for inclusion in Faculty Bibliography 2000 s by an authorized administrator of STARS. For more information, please contactSTARS@ucf.edu.

\section{Recommended Citation}

Wang, Haining and Zou, Shengli, "Extremely low scattering cross section of a perforated silver film" (2009). Faculty Bibliography 2000s. 2281.

https://stars.library.ucf.edu/facultybib2000/2281

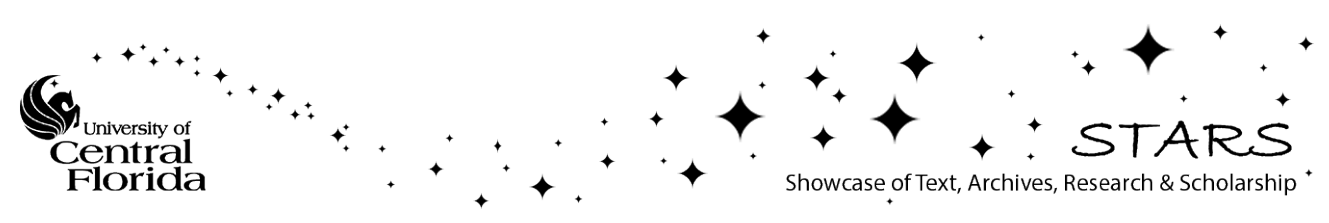




\section{Extremely low scattering cross section of a perforated silver film}

Cite as: Appl. Phys. Lett. 94, 073119 (2009); https://doi.org/10.1063/1.3086889

Submitted: 16 November 2008 . Accepted: 02 February 2009 . Published Online: 20 February 2009

Haining Wang, and Shengli Zou

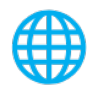

\section{Applied Physics Letters}

Mid-IR and THz frequency combs special collection 


\title{
Extremely low scattering cross section of a perforated silver film
}

\author{
Haining Wang and Shengli Zou ${ }^{\text {a) }}$ \\ Department of Chemistry, University of Central Florida, 4000 Central Florida Blvd., Orlando, Florida \\ 32816-2366, USA
}

(Received 16 November 2008; accepted 2 February 2009; published online 20 February 2009)

\begin{abstract}
With a perforated silver film, we demonstrate that an extremely low scattering cross section with an efficiency of less than $1 \%$ can be achieved at tunable wavelengths with tunable widths. The resonance wavelength, width, and intensity are influenced by the size, shape, and arrangement pattern of the holes, as well as the distance separating the holes along the polarization direction. The discovered phenomenon holds promise for the development of photovoltaic devices with improved energy conversion efficiency. (C) 2009 American Institute of Physics. [DOI: 10.1063/1.3086889]
\end{abstract}

When light irradiates a metal surface, the conduction electrons of the metal may interact with the incident electromagnetic wave and be excited. The collective excitations of the conduction electrons are called surface plasmons. ${ }^{1}$ Due to field confinement and enhancement, surface plasmons have been utilized in many disciplines. ${ }^{2-5}$ Ebbesen's pioneering work on the extraordinary optical transmission through subwavelength hole arrays ${ }^{6}$ stimulated extensive interest in investigating the optical properties of perforated metal films. ${ }^{7-11}$ This enhanced transmission has been explained due to the excitations of surface plasmons ${ }^{6-8}$ of the metal film, although other mechanisms have also been used in describing this phenomenon. ${ }^{9-11}$ Since an enhanced transmission indicates a reduced scattering at the same frequency, the scattering spectra of such films have attracted less attention.

Using the discrete dipole approximation (DDA) method, ${ }^{12}$ we demonstrate that a remarkably low scattering cross section of a perforated metal film can be achieved at tunable wavelengths with tunable widths. The designed film may be coated on a solar cell to improve its energy harvesting efficiency at desirable wavelengths. In the DDA method, the target particle is divided into an array of $N$ polarizable cubes (dipoles). By solving the resulting $3 N$ linear equations, the induced dipole of each cube can be obtained and the absorption, scattering, and extinction spectra of the particle can be calculated. A detailed description of the DDA method can be found in Draine's articles. ${ }^{12}$ Herein, we provide only the equations used for calculating the extinction and scattering cross sections of a particle, which are given as

$$
\begin{aligned}
C_{\text {ext }} & =\frac{4 \pi k}{\left|E_{0}\right|^{2}} \sum_{j=1}^{N} \operatorname{Im}\left(E_{\text {inc }, j}^{*} \cdot P_{j}\right), \\
C_{\text {sca }} & =\frac{k^{4}}{\left|E_{0}\right|^{2}} \int d \Omega\left|\sum_{j=1}^{N}\left[P_{j}-\hat{n}\left(\hat{n} \cdot P_{j}\right)\right] \exp \left(-i k \hat{n} \cdot r_{j}\right)\right|^{2},
\end{aligned}
$$

where $E_{0}$ is the amplitude of the incident wave, $k=2 \pi / \lambda$ is the wave vector at the incident wavelength $\lambda, \hat{n}$ is the unit vector along the scattering direction, $E_{\mathrm{inc}, j}$ and $P_{j}$ represent the incident electric field and induced dipole at the cube $j$ position, respectively, $r_{j}$ denotes the coordinate of cube $j$,

${ }^{a)}$ Electronic mail: szou@mail.ucf.edu. and $\Omega$ is the integration angle in the space. The absorption cross section is given by $C_{\mathrm{ext}}-C_{\mathrm{sca}}$. The scattering, absorption, and extinction cross sections obtained using Eqs. (1) and (2) are in units of area; their respective efficiencies are calculated by dividing them by the physical area of the particle or film.

In the simulations, the incident wave vector was taken to be parallel to the $X$ axis, and the polarization direction is taken to be along the $Z$ axis. The film was arranged in the $Y Z$ plane. The film thickness has a slight influence on the resonance wavelength and is set to equal $100 \mathrm{~nm}$ in all of the simulations. The dielectric constants of silver were taken from Palik's handbook. ${ }^{13}$ Periodic boundary conditions ${ }^{14}$ were applied to the DDA method.

We started with a silver film perforated with cylindrical holes that were arranged in a square lattice; the centers of nearest neighbor holes were separated by $400 \mathrm{~nm}$ from each other. The diameter of the holes was set at 100, 200, and $300 \mathrm{~nm}$ in the calculations. As shown in Fig. 1(a), when the distance between the hole centers was fixed at $400 \mathrm{~nm}$, the resonance dip became more prominent with increasing hole diameter. The width of the resonance dip also broadened correspondingly. For a film with $100 \mathrm{~nm}$ diameter holes, the scattering cross section at $470 \mathrm{~nm}$ resonance wavelength decreased only by $26 \%$ compared with that of the solid film. When the diameter of the hole was increased to $300 \mathrm{~nm}$, the resonance wavelength was redshifted to $570 \mathrm{~nm}$, and the scattering efficiency of the film dropped significantly to less than $1 \%$ of that observed with the solid film. The absorption spectra of the films depicted in Fig. 1(a) are shown in Fig. 1(b). These spectra indicate that the absorption peaks of the films can be observed at the same wavelength associated
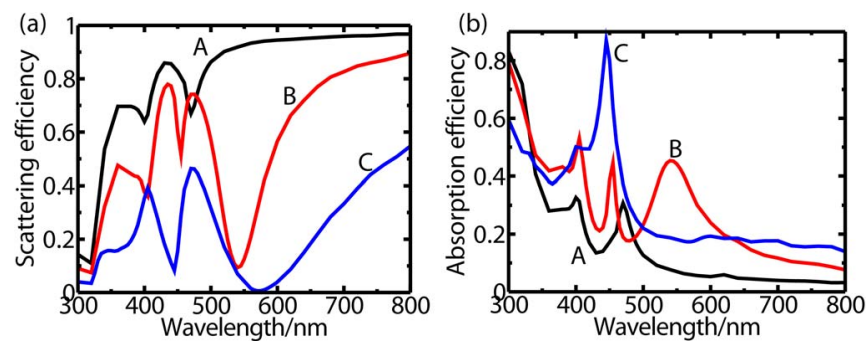

FIG. 1. (Color online) (a) Scattering and (b) absorption spectra for a $100 \mathrm{~nm}$ thick silver film with cylindrical holes with different diameters $(d)[(\mathrm{A})$ $d=100 \mathrm{~nm},(\mathrm{~B}) d=200 \mathrm{~nm}$, and (C) $d=300 \mathrm{~nm}$ ] arranged in a square lattice with a nearest neighbor separation of $400 \mathrm{~nm}$. 

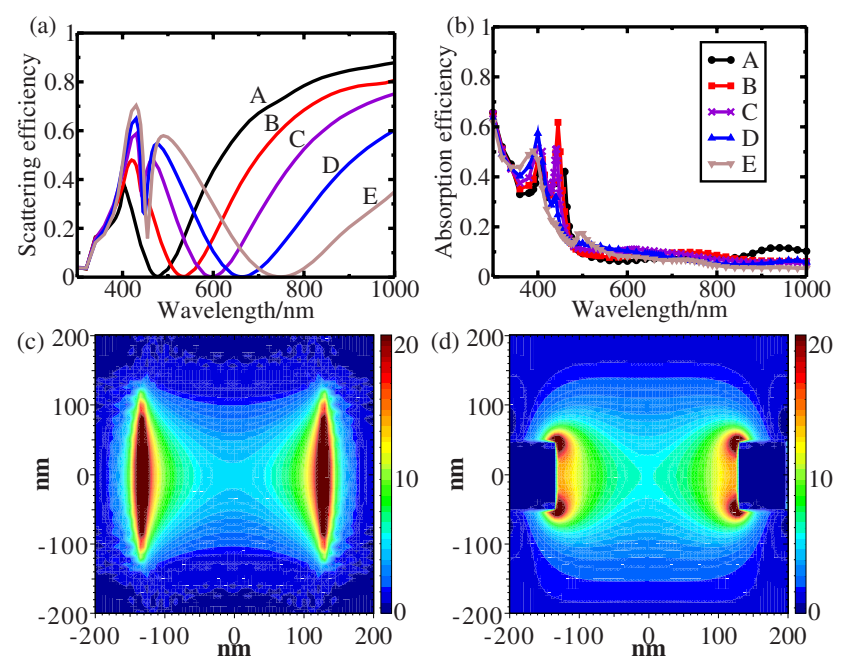

FIG. 2. (Color online) (a) Scattering and (b) absorption spectra of a $100 \mathrm{~nm}$ thick silver film with rectangular holes with a nearest neighbor separation of $400 \mathrm{~nm}$; (A) $220 \times 320 \mathrm{~nm}^{2}$, (B) $240 \times 290 \mathrm{~nm}^{2}$, (C) $260 \times 260 \mathrm{~nm}^{2}$, (D) $290 \times 240 \mathrm{~nm}^{2}$, and (E) $320 \times 220 \mathrm{~nm}^{2}$ (the latter number is the hole edge length parallel to the polarization direction). Electric field, $|E|^{2}$, contour plots at $595 \mathrm{~nm}$ wavelength in the (c) $Y Z$ and (d) $X Z$ planes for a film with $260 \times 260 \mathrm{~nm}^{2}$ holes are shown.

with a remarkably low scattering efficiency. The enhanced absorption indicates that the surface plasmon does play a role in the scattering reduction in the film.

We also investigated the effect of the shape and orientation of the holes on the scattering spectra of the film. In the simulations, rectangular holes with different aspect ratios (i.e., the ratio between the long and short edge lengths) were arranged in a square lattice with a nearest neighbor spacing of $400 \mathrm{~nm}$. The area of the holes was taken to be about $67600 \mathrm{~nm}^{2}$, which is approximately equivalent to the area of a cylindrical hole with a diameter of $300 \mathrm{~nm}$. The shape of the rectangular holes was chosen to be either 220 $\times 320 \mathrm{~nm}^{2}, 240 \times 290 \mathrm{~nm}^{2}$, or $260 \times 260 \mathrm{~nm}^{2}$, respectively. The holes were arranged so that either their short or long edge was parallel to the polarization direction. Figure 2(a) shows that an extraordinarily low scattering cross section can always be obtained with holes of different shapes and orientations. The resonance wavelength appears at short wavelength when the long edge of the rectangle is parallel to the polarization direction and shifts to red with decreasing edge length along the polarization direction. For example, when the $220 \times 320 \mathrm{~nm}^{2}$ rectangular holes were arranged with the $320 \mathrm{~nm}$ edge along the polarization direction, a reduced scattering dip appeared at $475 \mathrm{~nm}$ wavelength. The resonance wavelength was shifted to $750 \mathrm{~nm}$ when the $220 \mathrm{~nm}$ edge was arranged along the polarization direction. The dip width widened with decreasing edge length along the polarization direction. The corresponding absorption spectra of the films are displayed in Fig. 2(b), which indicates that the absorption spectra follow the same trend and are smaller in magnitude in comparison to the scattering spectra.

To understand the mechanism leading to the reduced scattering of the film, we calculated the electric field, $|E|^{2}$, around the holes. The electric field contour plots at $595 \mathrm{~nm}$ for films with $260 \times 260 \mathrm{~nm}^{2}$ holes are displayed in Figs. 2(c) and 2(d). Figure 2(c) shows the electric field contour plot in the $Y Z$ plane, $5 \mathrm{~nm}$ from the film surface and facing the incident wave. Figure 2(d) displays the electric field con-
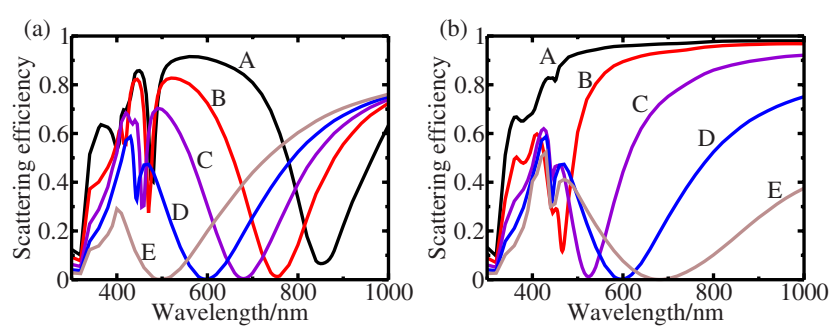

FIG. 3. (Color online) Scattering spectra of a $100 \mathrm{~nm}$ thick silver film with rectangular holes with a nearest neighbor separation of $400 \mathrm{~nm}$ and different edge lengths; (a) holes with the same edge length $(260 \mathrm{~nm})$ perpendicular to the polarization direction and with different parallel edge lengths; (b) holes with the same parallel edge length $(260 \mathrm{~nm})$ and with different perpendicular edge lengths. (A) $65 \mathrm{~nm}$, (B) $130 \mathrm{~nm}$, (C) $195 \mathrm{~nm}$, (D) $260 \mathrm{~nm}$, and (E) $325 \mathrm{~nm}$ for both (a) and (b).

tour plot in the $X Z$ plane through a hole center. The enhanced local electric field indicates that surface plasmons are excited at the wavelength associated with the reduced scattering. The highest enhanced electric field, $|E|^{2}$, obtained was 46. Figure 2(d) shows that the enhanced electric fields are locally confined around the hole area.

To further investigate the influence of the hole shape on the scattering spectra of the film, we fixed the edge length of the rectangular holes perpendicular to the polarization direction to be $260 \mathrm{~nm}$ and varied the other edge length from 65 to $325 \mathrm{~nm}$. The holes were arranged in a square lattice with a nearest neighbor separation of $400 \mathrm{~nm}$. Figure 3(a) shows that remarkably low scattering efficiencies could always be obtained when the edge length along the polarization direction was changed from 65 to $325 \mathrm{~nm}$. The resonance wavelength was shifted from 850 to $490 \mathrm{~nm}$ when the edge length was varied from 65 to $325 \mathrm{~nm}$. The simulations show that the lowest scattering efficiency grew larger than $10 \%$ when the edge length was decreased to $50 \mathrm{~nm}$.

The effect of the edge length perpendicular to the polarization direction was explored by keeping the parallel edge length set at $260 \mathrm{~nm}$ and varying the perpendicular edge length from 65 to $325 \mathrm{~nm}$. Figure 3(b) shows that the change in the perpendicular edge length has a significant influence on the scattering intensity of the film. For holes with a $325 \mathrm{~nm}$ edge length, a broad resonance dip with a low scattering efficiency of less than $1 \%$ was obtained at $680 \mathrm{~nm}$ wavelength. With decreasing edge length, the resonance wavelength was shifted to shorter wavelengths, the resonance width narrowed, and the lowest scattering efficiency increased substantially. When the edge length was decreased to $65 \mathrm{~nm}$, the resonance dip at $375 \mathrm{~nm}$ wavelength became very weak.

In the above simulations, the holes were always arranged in a square lattice and their center to center distance was kept at $400 \mathrm{~nm}$. To investigate the effects of the hole distance on the scattering efficiency and resonance wavelength, we arranged holes in a rectangular lattice. The dimension of the rectangular holes was kept to be $260 \times 260 \mathrm{~nm}^{2}$. We first fixed the hole distance perpendicular to the polarization direction $\left(D_{v}\right)$ to be $400 \mathrm{~nm}$ and then changed the parallel distance $\left(D_{p}\right)$ from 300 to $500 \mathrm{~nm}$. Figure 4(a) shows that the resonance wavelength was very sensitive to the hole spacing along the polarization direction. When $D_{p}$ was $300 \mathrm{~nm}$, the lowest scattering efficiency could be found at $455 \mathrm{~nm}$ wavelength. When $D_{p}$ was extended to $500 \mathrm{~nm}$, the resonance wavelength was shifted to $695 \mathrm{~nm}$ and the depth and width 

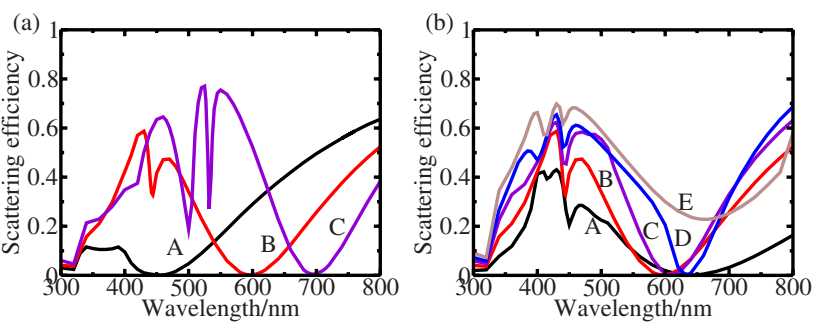

FIG. 4. (Color online) Scattering spectra for a $100 \mathrm{~nm}$ thick silver film with square holes of the same area but with different spacings. (a) Holes with a fixed $400 \mathrm{~nm}$ distance perpendicular to the polarization direction and with different parallel distances: (A) $300 \mathrm{~nm}$, (B) $400 \mathrm{~nm}$, and (C) $500 \mathrm{~nm}$. (b) Holes with a fixed $400 \mathrm{~nm}$ parallel distance and with different perpendicular distances: (A) $300 \mathrm{~nm}$, (B) $400 \mathrm{~nm}$, (C) $500 \mathrm{~nm}$, (D) $600 \mathrm{~nm}$, and (E) 800 nm.

of the resonance dip varied slightly. The observed distance dependence of the resonance wavelength was due to the propagation direction of the surface plasmon on the metal film. The excited surface plasmon around each hole propagates along the polarization direction, and the hole distance along the polarization direction is crucial in determining the resonance wavelength. The distance dependence of the resonance wavelength also indicates that the observed scattering reduction is not due to the coupling of diffracted waves.

We further fixed the hole spacing along the polarization direction $\left(D_{p}\right)$ to be $400 \mathrm{~nm}$ and varied the distance along the perpendicular direction $\left(D_{v}\right)$. Figure 4(b) shows that the resonance wavelength was not very sensitive to the perpendicular distance, but the depth of the resonance dip could be dramatically altered. With increasing $D_{v}$, the resonance dip became shallower due to the weaker coupling between the holes and smaller total hole area of the film.

In summary, we investigated the reduced scattering of a perforated silver film. Extraordinarily low scattering cross sections with efficiency close to zero at tunable wavelengths with tunable widths were obtained. The resonance wavelength, width, and intensity were all influenced by the size, shape, and arrangement pattern of the holes. For holes with the same area, rectangular holes with short edge length (larger than $100 \mathrm{~nm}$ ) along the polarization direction generated a more significant scattering reduction. The resonance wavelength shifted to red with decreasing edge length along the polarization direction; it also shifted to red as the distance between the holes along the polarization direction increased.

This work was supported by the ACS Petroleum Research Fund No. 48268-G6 and NSF CBET 0827725 Fund.

${ }^{1}$ R. H. Ritchie, Phys. Rev. 106, 874 (1957).

${ }^{2}$ S. Pillai, K. R. Catchpole, T. Trupke, and M. A. Green, J. Appl. Phys. 101, 093105 (2007).

${ }^{3}$ H. Ditlbacher, J. R. Krenn, B. Lamprecht, A. Leitner, and F. R. Aussenegg, Opt. Lett. 25, 563 (2000).

${ }^{4}$ K. Tanaka and M. Tanaka, Appl. Phys. Lett. 82, 1158 (2003).

${ }^{5}$ J. Pendry, Science 285, 1687 (1999).

${ }^{6}$ T. W. Ebbesen, H. J. Lezec, H. F. Ghaemi, T. Thio, and P. A. Wolff, Nature (London) 391, 667 (1998).

${ }^{7}$ H. J. Lezec, A. Degiron, E. Devaux, R. A. Linke, L. Martin-Moreno, F. J. Garcia-Vidal, and T. W. Ebbesen, Science 297, 820 (2002).

${ }^{8}$ S.-H. Chang, S. K. Gray, and G. C. Schatz, Opt. Express 13, 3150 (2005).

${ }^{9}$ M. M. J. Treacy, Phys. Rev. B 66, 195105 (2002).

${ }^{10}$ Q. Cao and P. Lalanne, Phys. Rev. Lett. 88, 057403 (2002).

${ }^{11}$ H. J. Lezec and T. Thio, Opt. Express 12, 3629 (2004).

${ }^{12}$ B. T. Draine and P. J. Flatau, "User guide for the discrete dipole approximation code DDSCAT.6.0," http://arXiv.org/abs/0809.0337v4.

${ }^{13}$ E. D. Palik, Handbook of Optical Constants of Solids (Academic, New York, 1985).

${ }^{14}$ E. M. Hicks, S. Zou, G. C. Schatz, K. G. Spears, R. P. Van Duyne, L. Gunnarsson, T. Rindzevicius, B. Kasemo, and M. Kall, Nano Lett. 5, 1065 (2005). 